\title{
Based on Difference Method of the Light Color Image Motion Target Recognition Technology
}

\author{
Wang Fang \\ Yangtze River College \\ East China Institute of Technology \\ Fuzhou, JX ,China \\ wtwfok@126.com
}

\author{
Li Peng \\ Vacotional and Technical School \\ East China Institute of Technology \\ Fuzhou, JX, China \\ pli@ecit.edu.cn
}

\begin{abstract}
Color images with light contain rich information, so there is too much interference to get the moving target detection. How to eliminate the effect of light in color image processing for moving target detection and recognition? It proposes a new moving target detection technology based on difference method. This method is based on normal background difference image method. It improves threshold settings with binary and filter processing to gain the more effective background image segmentation results. It is realized in $\mathrm{VC}++$ platform thorough the actual scene image simulation experiments. The results prove that the technology is feasible and effective and a small amount of calculation and easy to implement.
\end{abstract}

Keywords-moving target detection, difference method, threshold, image segmentation

\section{INTRODUCTION}

With the rapid development of computer, the color image is widely used in many fields. so to detect and recognize the real goal area in the color image is a research hot spot. The moving target detection technology in scene image is to use image processing algorithm to automatically detect and identify moving target from clutter background and strong noise environment. The information of color image is richer than gray image, so it's more difficult to gain information because of the influence of lighting factors such as fire. There are many algorithms of moving target detection which are divided by two: single frame image detection and image sequence detection. This article researches on single frame image. This detection is to use the gray information of the single frame image to separate moving target and background. The effect of this separating is depended on character of moving target, background and applying environment. Furthermore, it relies on the identify ability of moving target and background. At first it is supposed as combination of background image, noise image and moving target image. Then there are two steps that first to reprocess single frame image and second to segment threshold. There are four methods to detect moving target such as template matching, optical flow, time difference and background deduction. Template matching needs to use the fixed template. Optical flow has the bad resistance to dry so it is not suitable for complicated background. Time difference can't accurately get the moving target. So the background deduction is a technology to use the difference of current image and background image to detect moving target. The advantage is that it computes simple, runs fast and it can provide the complete object information. This article studies the algorithm of detecting moving target in static color image.

\section{TECHNOLOGY IMPROVEMENT}

The early image processing uses to improve the quality of image to improve people's visual effect. The common image processing methods contains image transform, image enhance, image restore, image coding, image segmentation, image comprehension and recognition. The algebraic operation of image is to get out image by point-to-point arithmetic operation on two input images. If input images as $\mathrm{A}(\mathrm{x}, \mathrm{y})$ and $\mathrm{B}(\mathrm{x}, \mathrm{y})$, output image as $\mathrm{C}(\mathrm{x}, \mathrm{y})$, then arithmetic operation expression is as follows:

$$
C(x, y)=A(x, y)+B(x, y) ; C(x, y)=A(x, y)-B(x, y)
$$

$$
\mathrm{C}(\mathrm{x}, \mathrm{y})=\mathrm{A}(\mathrm{x}, \mathrm{y}) \times \mathrm{B}(\mathrm{x}, \mathrm{y}) ; \mathrm{C}(\mathrm{x}, \mathrm{y})=\mathrm{A}(\mathrm{x}, \mathrm{y}) / \mathrm{B}(\mathrm{x}, \mathrm{y})
$$

Image subtract is usually applied in change and motion detection, so is also named as image difference operation. Under the controlled environment, the background is considered fixed, so it is directly used to detect change and motion of object. This method combines Yan value processing to be one of the most effective establishing machine visual systems. In a relative stable environment, it is supposed that the background changes slowly and meets certain distribution, then it establish background model and apply difference method to detect moving object.

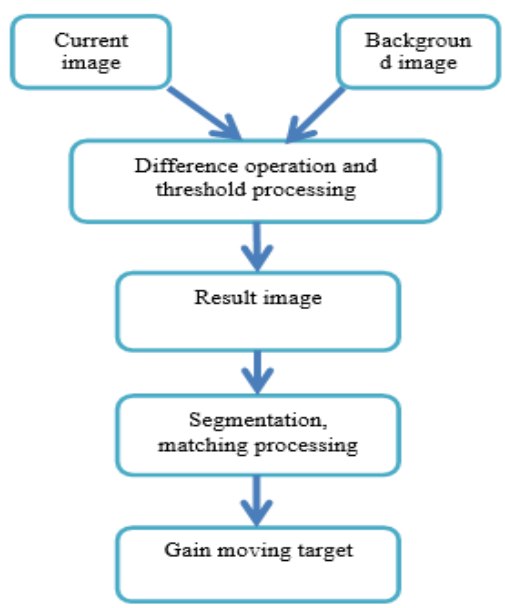

Fig.1 difference method detect moving target flow chart 
Difference method actually is subtraction operation of images. It is subtraction of images that the same scene in different time or the same scene in different band. Different images supply the difference information between images which can guide dynamic testing, moving object detect, image background eliminate and moving target identify. Its operation flow chart is shown in Fig. 1.

There is obvious effect to apply difference technology to eliminate image background which is better in image with fire than in common image. At first, this algorithm store the background image, because there are differences between moving object and background in gray or color, then subtract background image and current image. Every pixel value in subtraction is compared with a predefined threshold If the pixel value is larger than threshold, it is fore point, otherwise it is background point. Its basic operation flow chart is shown in Fig. 2.

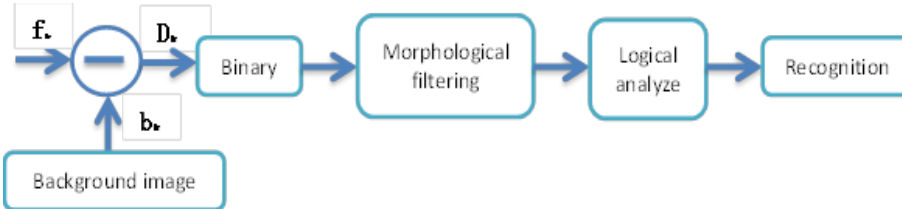

Fig.2 difference method principle flow chart

At first, it uses Equation (1) to compute the difference of background image $b_{k}$ and current image $f_{x}$. Then it processes difference image binary and morphological filtering, and analyzes the regional connectivity for the result $\mathrm{R}_{\mathrm{k}}$. If any connectivity region area larger than the certain threshold, then this region will be the detecting target and regional context, so it can certain the smallest bounding rectangle of the target.

$$
D_{k}(x, y)=\left|f_{k}(x, y)-b_{k}(x, y)\right|
$$

$f_{k}(x, y)$ is the current image, $b_{k}(x, y)$ is the background image, $D_{k}(x, y)$ is the difference image.

$$
R_{k}(x, y)\left\{\begin{array}{lll}
0 & \text { Background } & D_{k}(x, y)>T \\
1 & \text { Foreground } & D_{k}(x, y) \leq T
\end{array}\right.
$$

$\mathrm{T}$ is the binary setting threshold.

There are some focus questions as follows when using this algorithm:

1) How to gain the background image? It is required not have moving target, but in fact it is hard to meet this precision request especially for image including fire with light complex effect.

2) How to eliminate the complex information in background and how to dry process? The ideal condition is that there is no change and noise pollution in background and current images, but it is hard to realize in fact.

How to select threshold? It directly decides the detecting result is good or bad. Only adapt threshold can segment the area with moving target. So we can select threshold many times by experience and then optimize the selection.

Next the basic operation of mathematical morphology can be applied to binary image. Through combination, some morphological analysis algorithms and a series of morphological processing algorithms are formed including expansion, corrosion, open, close and other operations. Mathematical morphology defines two of the most basic operations called corrosion and expansion. In this paper, corrosion calculation is used for morphological treatment.

Corrosion refers to the detection of an image with a "probe" (that is, a primitive or structural element of a certain shape), so as to find the region within the image where the primitive can be placed. It is a process of eliminating boundary points and making the boundary shrink inward which can be used to eliminate small and meaningless objects. The implementation of corrosion is also based on the concept of filling structural elements. The process of filling with structural elements depends on a basic concept of Euclidean space -- translation. So let's label A set A shifted by some distance along the vector $\mathrm{x}$.

$$
A_{x}=\{a+x: a: A\}
$$

Set $\mathrm{A}$ is corroded by $\mathrm{B}$, denoted as $A \Theta B$, and defined as Equation (4).

$$
A \Theta B=\{x: B+x: A\}
$$

Where $\mathrm{A}$ is called the input image and $\mathrm{B}$ is called the structural element. $\mathrm{A} \Theta_{\mathrm{B}}$ is made up of all the points $\mathrm{x}$ that you can shift $B$ by $x$ and still include in $A$. If you think of $B$ as $A$ template, then $A \Theta_{B}$ is composed of the origin of all the templates that can be filled into A during the translation of the template. According to the relationship between the origin and the position of structural elements, the corroded image can be roughly divided into two categories.

1) If the origin is inside the structural element, the etched image is a subset of the input image, as shown in figure 3 .

2) If the origin is outside the structural element, the corroded image may no longer input the interior of the image, as shown in Figure 4.

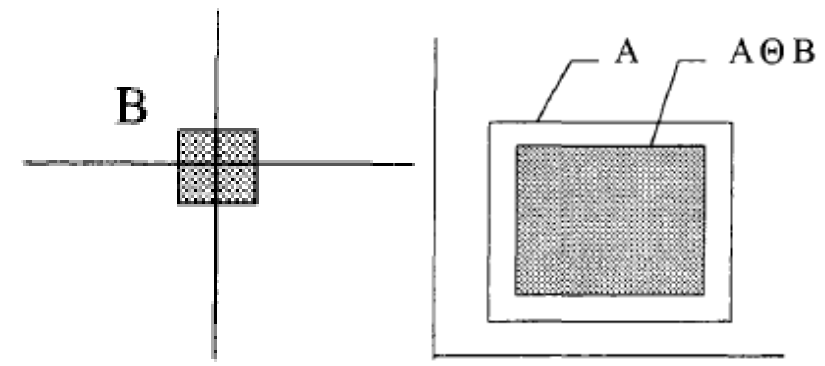

Fig.3 corrosion is analogous to shrinkage
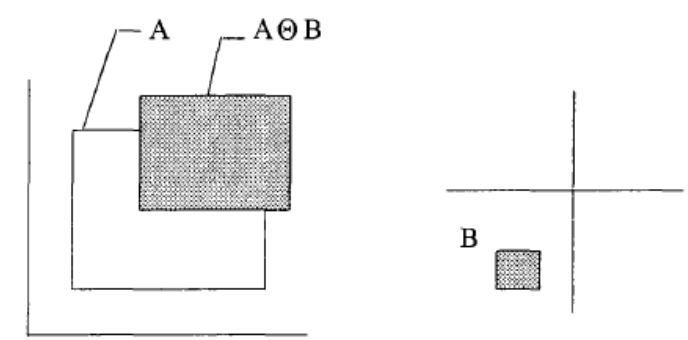

Fig.4 corrosion is not a subset of the input image 
In addition to the filling form, corrosion is also expressed in a more important form shown in Equation (5).

$$
\mathrm{A} \ominus \mathrm{B}=\cap\{\mathrm{A}-\mathrm{b}: \mathrm{b} \in \mathrm{B}\}
$$

Corrosion can be obtained by translating the input image to -b(which belongs to the structural element) and calculating the intersection of all the translations.

Expansion satisfies two fundamental relations of operation, namely, commutative law and associative law. That is Equation (6).

$$
\mathrm{A} \oplus \mathrm{B}=\mathrm{B} \oplus \mathrm{A}
$$

$$
\mathrm{A} \oplus(\mathrm{B} \oplus \mathrm{C})=(\mathrm{A} \oplus \mathrm{B}) \oplus \mathrm{C}
$$

According to Equation (7), corrosion operation is not commutative, but corrosion operation has associative law.

$$
\mathrm{A} \Theta(\mathrm{B} \oplus \mathrm{C})=(\mathrm{A} \Theta \mathrm{B}) \Theta \mathrm{C}=(\mathrm{A} \Theta \mathrm{C}) \Theta \mathrm{B}
$$

This formula can be shown that when image $\mathrm{A}$ is corroded by A large structural element $\mathrm{B} \oplus \mathrm{C}$, the result is the same as that of continuous corrosion by $\mathrm{B}$ and $\mathrm{C}$, and the corrosion result is independent of the corrosion order of structural elements B and C. According to this property, we can only store some simple and basic structural elements B, $\mathrm{C}$ and so on. Once needed, they can continuously corrode the image to replace various complex structural elements.

Experimental results show that the size and shape of structural elements have different effects on the results of mathematical morphological operations. We can see that some new and effective ideas and methods have been generated with the support of basic operations of mathematical morphology, and their practical applications have opened up quite attractive fields. This also proves the vitality of mathematical morphology. Computer simulation experiments show that the results of image processing based on mathematical morphology are more suitable for visual information processing and analysis.

\section{EXPERIMENTAL RESULTS AND ANALYSIS}

According to the above algorithm chart, we use $\mathrm{VC}++$ to test as follows.
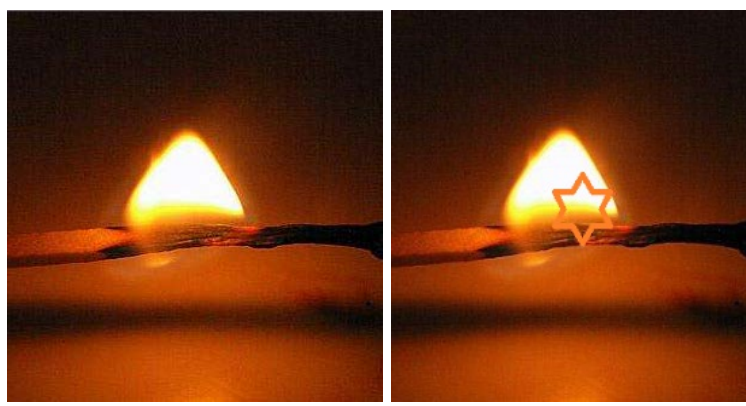

Fig.5 current image

Fig.6 background image

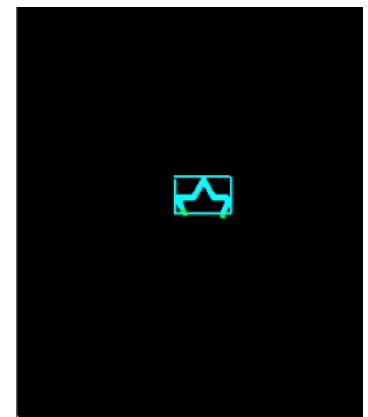

Fig.7 difference image

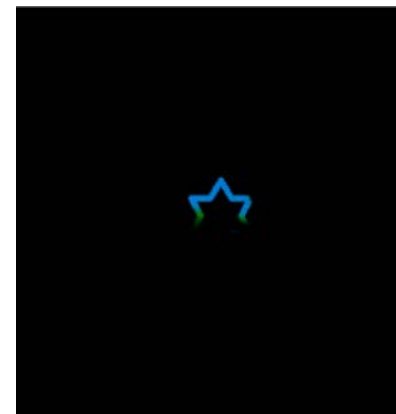

Fig.8 tag motion area
The above images show the test result, Fig. 5 shows the current image $f_{x}$, Fig. 6 shows the background image $b_{k}$, Fig. 7 shows the difference image between current image and background image, Fig. 8shows the binary image after threshold segment, Fig. 8 shows the tag motion area after filter processing. The detecting area with whole motion target has been shown in Fig. 8. although the connection of motion target outline and area can be preserved exactly, there are some sensitive factors like light impacting on judging and identifying target area.

\section{SUMMARY}

This article mainly studies reasonable binary image after threshold segmentation basing on motion target detection and recognition by difference in color image including fire complex information, and morphological filtering process, then more exactly detecting on motion target area. After many trials, this algorithm is fast and feasible. It computes simple and has advantage in inhibition of complex background and noise processing. This technology can be actually applied in text image processing, remote sensing and biomedical image analysis, security monitoring, traffic monitoring and so on.

\section{ACKNOWLEDGEMENT}

Project fund no : GJJ151539

\section{REFERENCES}

[1] Cui Yi. Image process and analysis. Morphological method and application[ M]. Bei Jing : Science Press, 2000 :127 131.

[2] Peng Jiaxiong, Zhou wenlin. Infrared background suppression and small motion target segment detect [ J]. Electronic journal, 1999 , 27(12):47 51.

[3] Gao Yinghui, Li Jicheng, Shen Zhenkang. Research on reprocess method of Infrared target detect[ J].

[4] Infrared and laser engineering, 2004, 33(2), 154-158.

[5] Xu Ying. Research on method of infrared little target detect [ J]. IR magazine.

[6] Zhang Yujin. Image segmentation[M]. Bei Jing : Science Press, 2001. 